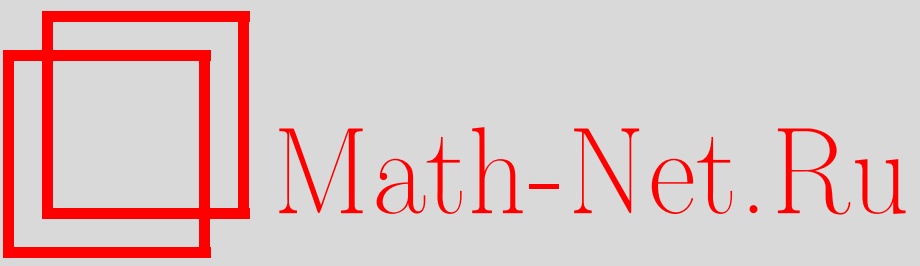

М. В. Шамолин, Пространственные топографические системы Пуанкаре и системы сравнения, УМH, 1997, том 52, выпуск 3, 177-178

DOI: https://doi.org/10.4213/rm859

Использование Общероссийского математического портала Math-Net.Ru подразумевает, что вы прочитали и согласны с пользовательским соглашением

http://www.mathnet.ru/rus/agreement

Параметры загрузки:

IP: 3.89.197.203

26 апреля 2023 г., 17:28:55 


\title{
ПРОСТРАНСТВЕННЫЕ ТОПОГРАФИЧЕСКИЕ СИСТЕМЫ ПУАНКАРЕ И СИСТЕМЫ СРАВНЕНИЯ
}

\author{
М. В. ШАмолин
}

1. Топографические системы в плоском случае. Еще Пуанкаре предложил метод отыскания замкнутых орбит дифференциального уравнения на плоскости [1]. Для этого ему потребовалось ввести понятие "топограбиической системы" (ТСП) [2].

В его трудах впервые начала рассматриваться достаточно гладкая алгебраическая функция двух переменных, которая стремится к бесконечности, когда одна из ее переменных стремится к бесконечности, положительно определена в окрестности начала координат, имеет первые производные, обращающиеся в нуль также лишь в начале координат, обладает тем свойством, что кривая ее контактов с некоторым векторным полем имеет в начале координат изолированную особую точку (о кривых контактов см. [2], [3]).

При выполнении последних условий поверхности уровня рассматриваемой функции дают так называемую топографическую систему вложенных друг в друга кривых, имеющую вершину в начале.

Обнаружение замкнутых траекторий происходит следующим образом. Вычисляя угол между векторами поля, образующими семейство ТСП, и векторами исследуемого поля динамической системы, можно получить информацию о расположении траекторий исследуемого векторного поля. Чтобы доказать отсутствие замкнутых фазовых характеристик достаточно показать, что вьполнено неравенство

$$
\left.\left(v_{1}, v_{2}\right)\right|_{\mathbb{R}^{2}} \geqslant 0
$$

Здесь $v_{1}$ - векторное поле, касательное к ТСП, $v_{2}$ - векторное поле динамической системш.

2. Характеристические функции и кривые контактов векторных полей в плоском случае. Системы сравнения. С понятием ТСП тесно связано понятие характеристической функции двух полей на плоскости. Последняя функция определяет кососимметрическую форму. Если $F\left(x_{1}, x_{2}\right)=$ const есть семейство замкнутых кривых, то система, имеющая явный вид гамильтоновой, $x_{1}^{\prime}=-\frac{\partial F}{\partial x_{2}}, x_{2}^{\prime}=\frac{\partial F}{\partial x_{1}}$, задает векторное поле, касательное к семейству кривых ТСП. Тогда последнее неравенство эквивалентно $X_{1} Y_{2}-X_{2} Y_{1} \geqslant 0$, в котором $Y_{1}=-\frac{\partial F}{\partial x_{2}}, Y_{2}=\frac{\partial F}{x_{1}}$ есть некоторое векторное поле. Оно касается кривых ТСП.

Рассмотрим две системы уравнений на плоскости. Эти уравнения задаются полями $X=$ $\left\{X_{1}, X_{2}\right\}$ и $Y=\left\{Y_{1}, Y_{2}\right\}$ в некоторых координатах. Естественно рассмотреть функцию $\chi=X_{1} Y_{2}-X_{2} Y_{1}$, которая отвечает за знак синуса угла между полями $X$ и $Y$. Очевидно, что $\chi=0$ там и только там, где поля $X$ и $Y$ касаются.

Функция $\chi$ удовлетворяет следующим свойствам: $\chi(X, Y)=-\chi(Y, X), \chi(\lambda X, Y)=\lambda \chi(X, Y)$ для любой действительной функции $\lambda$.

ОПРЕДЕЛЕнИЕ. Функцию $\chi$ мы назовем характеристической функцией двух векторных полей, а уравнение $\chi=0$ назовем уравнением кривой контактов для полей $X$ и $Y$.

Вычислять характеристическую функцию, конечно, можно для любых двух векторных полей на плоскости. В этой связи назовем системой сравнения для данной системы ту систему, качественное расположение траекторий которой полностью известно.

В работе [3] приведены теоремы, уточняющие некоторые методы Пуанкаре в данном вопросе.

3. Топографические системы в пространственном случае. Пространственные ТСП можно определить аналогично ТСП на плоскости (см. выше). При этом (невырожденные) гиперповерхности уровня коразмерности 1 в пространстве $\mathbb{R}^{n}$ образуют топографическую систему вложенных друг в друга гиперповерхностей, имеющих вершину в особой точке. 
Теорема. Пусть в односвязной области $D$ пространства $\mathbb{R}^{n}$, содерэсащей единственную точку покоя $x_{0}$ достаточно гладкого векторного поля $v_{1}$, существует гиперповерхность $\Gamma \ni x_{0}$, пересекающая граничу $\partial D$ по поверхности $\gamma$ (поверхность $\gamma$ может быть бесконечно удалена), такая, что существует ТСП с центром в $x_{0}$, задаваемая гладкой функцией $V$, продолжающаяся вдоль Г до $\gamma$, заполняющая область $K \subseteq D$ и обладающая свойством (1) (вместо $\mathbb{R}^{2}$ следует писать $\mathbb{R}^{n}, v_{2}=\operatorname{grad} V$ ) почти всюду в $K$ за исключением, быть может, некоторых гиперповерхностей, не содержащих внутри себя $x_{0}$. (Здесь $V=$ const - семейство гиперповерхностей ТСП.)

Тогда во всей области $D$ не существует ни одной замкнутой кривой, состоящей из траекторий поля $v_{1}$ и пересекающей $\Gamma$.

Напомним, что пространственные ТСП особенно удачно помогают решить в ряде случаев проблему различения центра и фокуса. В последнем случае вовсе не обязательно иметь с центром в данной особой точке ТСП. Система сравнения может иметь либо притягивающую, либо отталкивающую особую точку.

Пусть в области $D$, содержащей единственную особую точку системы (А), заданной для простоты на плоскости, стоит проблема различения центра и фокуса. Пусть в этой же области система (Б) имеет ту же единственную особую точку $x_{0}$.

Характеристическая функция в пространственном случае строится следующим образом. Пусть $v_{1}, v_{2}-$ два гладких векторных поля в пространстве $\mathbb{R}^{n}$. По полю $v_{1}$ строится (неоднозначно) нормальное гладкое векторное поле $n$. В каждом конкретном случае поле $n$ строится из тех же соображений, которые позволяют получить в дальнейшем знакоопределенную характеристическую функцию. Последняя определяется как скалярное произведение

$$
\chi=\left(n, v_{2}\right) .
$$

ПрЕДЛОЖЕНИЕ. Пусть область D является притягиваемой (отталкиваемой) точкой $x_{0}$ системы (Б). Тогда если характеристическая функиия $\chi((A),($ Б)) положительно (отрицательно) определена в области $D$, то область $D$ является притягиваемой (отталкиваемой) точкой $x_{0}$ системы (A).

4. Четномерный случай. В четномерном случае характеристическая функция имеет наиболее естественньй вид.

ПримеР. Рассмотрим систему слабо перевязанных маятников, т.е. консервативную систему с мальми (неконсервативными) добавками, задаваемую полем $\left\{X_{1}, X^{1}, \ldots, X_{n}, X^{n}\right\}$ в координатах $x=\left\{x_{1}, x^{1}, \ldots, x_{n}, x^{n}\right\}$ следующего вида: $X_{i}=-x^{i}+\varepsilon F_{i}(x), X^{i}=G_{i}(x)+\varepsilon F^{i}(x)$, $d G(0) \geqslant 0$. Тогда естественно выбрать характеристическую функцию в виде

$$
\chi=\sum_{i=1}^{n}\left(X_{i} Y^{i}-X^{i} Y_{i}\right)
$$

где векторное поле $Y$ системы сравнения имеет следующий вид: $Y=\left\{Y_{1}, Y^{1}, \ldots, Y_{n}, Y^{n}\right\}$, где $Y_{i}=-x^{i}, Y^{i}=G_{i}(x)$.

\section{СПИСОК ЛИТЕРАТУРЫ}

[1] Пуанкаре А. О кривых, определяемых дифференциальньми уравнениями. М.-Л.: ОГИЗ, 1947. [2] Пуанкаре А. Новые методы в небесной механике // Пуанкаре А. Избранные труды. Т. 1, 2. М.: Наука, 1971, 1972. [3] Шамолин М. В. // Вестн. МГУ. Сер. 1. Матем., мех. 1993. № 2. C. $66-70$.

Московский государственньй

Принято редколлегией университет им. М.В. Ломоносова 06.06.1996 